\title{
Rees algebras and polyhedral cones of ideals of vertex covers of perfect graphs
}

\author{
Rafael H. Villarreal
}

Received: 6 August 2006 / Accepted: 12 July 2007 / Published online: 10 August 2007

(C) Springer Science+Business Media, LLC 2007

\begin{abstract}
Let $G$ be a perfect graph and let $J$ be its ideal of vertex covers. We show that the Rees algebra of $J$ is normal and that this algebra is Gorenstein if $G$ is unmixed. Then we give a description-in terms of cliques-of the symbolic Rees algebra and the Simis cone of the edge ideal of $G$.
\end{abstract}

Keywords Perfect graphs · Normality · Edge ideals · Symbolic Rees algebras · Standard Gorenstein algebras · Max-flow min-cut · Clutters · Simis cone $\cdot$ Hilbert basis - Totally dual integral

Mathematics Subject Classification (2000) Primary 13H10 - Secondary 13F20 . $13 \mathrm{~B} 22 \cdot 52 \mathrm{~B} 20$

\section{Introduction}

Let $R=K\left[x_{1}, \ldots, x_{n}\right]$ be a polynomial ring over a field $K$ and let $I$ be an ideal of $R$ of height $g \geq 2$ minimally generated by a finite set $F=\left\{x^{v_{1}}, \ldots, x^{v_{q}}\right\}$ of squarefree monomials of degree at least two. As usual we use $x^{a}$ as an abbreviation for $x_{1}^{a_{1}} \cdots x_{n}^{a_{n}}$, where $a=\left(a_{1}, \ldots, a_{n}\right) \in \mathbb{N}^{n}$. A clutter $\mathcal{C}$ with vertex set $X$ is a family of subsets of $X$, called edges, none of which is included in another. The set of vertices and edges of $\mathcal{C}$ are denoted by $V(\mathcal{C})$ and $E(\mathcal{C})$ respectively. We can associate to the ideal $I$ a clutter $\mathcal{C}$ by taking the set of indeterminates $X=\left\{x_{1}, \ldots, x_{n}\right\}$ as vertex set and $E=\left\{S_{1}, \ldots, S_{q}\right\}$ as edge set, where $S_{k}$ is the support of $x^{v_{k}}$, i.e., $S_{k}$ is the set of variables that occur in $x^{v_{k}}$. For this reason $I$ is called the edge ideal of $\mathcal{C}$. To stress

Partially supported by CONACyT grant 49251-F and SNI, México.

R.H. Villarreal $(\bowtie)$

Departamento de Matemáticas, Centro de Investigación y de Estudios Avanzados del IPN, Apartado

Postal 14-740, 07000 Mexico City, DF, Mexico

e-mail: vila@math.cinvestav.mx 
the relationship between $I$ and $\mathcal{C}$ we will use the notation $I=I(\mathcal{C})$. The $n \times q$ matrix with column vectors $v_{1}, \ldots, v_{q}$ will be denoted by $A$. It is called the incidence matrix of $\mathcal{C}$. It is usual to call $v_{i}$ the incidence vector or characteristic vector of $S_{i}$.

The blowup algebras studied here are: (a) the Rees algebra

$$
R[I t]=R \oplus I t \oplus \cdots \oplus I^{i} t^{i} \oplus \cdots \subset R[t]
$$

where $t$ is a new variable, and (b) the symbolic Rees algebra

$$
R_{S}(I)=R \oplus I^{(1)} t \oplus \cdots \oplus I^{(i)} t^{i} \oplus \cdots \subset R[t],
$$

where $I^{(i)}$ is the $i$ th symbolic power of $I$.

The Rees cone of $I$, denoted by $\mathbb{R}_{+}(I)$, is the polyhedral cone consisting of the non-negative linear combinations of the set

$$
\mathcal{A}^{\prime}=\left\{e_{1}, \ldots, e_{n},\left(v_{1}, 1\right), \ldots,\left(v_{q}, 1\right)\right\} \subset \mathbb{R}^{n+1},
$$

where $e_{i}$ is the $i$ th unit vector. It is well documented [9-11] that Rees cones are an effective device to study algebraic and combinatorial properties of blowup algebras of square-free monomial ideals and clutters. They will play an important role here (Lemma 2.3). The normalization of $R[I t]$ can be expressed in terms of Rees cones as we now explain. Let $\mathbb{N} \mathcal{A}^{\prime}$ be the subsemigroup of $\mathbb{N}^{n+1}$ generated by $\mathcal{A}^{\prime}$, consisting of the linear combinations of $\mathcal{A}^{\prime}$ with non-negative integer coefficients. The Rees algebra of $I$ can be written as

$$
R[I t]=K\left[\left\{x^{a} t^{b} \mid(a, b) \in \mathbb{N} \mathcal{A}^{\prime}\right\}\right]
$$

According to [20, Theorem 7.2.28] the integral closure of $R[I t]$ in its field of fractions can be expressed as

$$
\overline{R[I t]}=K\left[\left\{x^{a} t^{b} \mid(a, b) \in \mathbb{Z}^{n+1} \cap \mathbb{R}_{+}(I)\right\}\right] .
$$

Hence, by Eqs. (1) and (2), we get that $R[I t]$ is a normal domain if and only if the following equality holds:

$$
\mathbb{N} \mathcal{A}^{\prime}=\mathbb{Z}^{n+1} \cap \mathbb{R}_{+}(I) .
$$

In geometric terms this means that $R[I t]=\overline{R[I t]}$ if and only if $\mathcal{A}^{\prime}$ is an integral Hilbert basis, that is, a Hilbert basis for the cone it generates. Rees algebras and their integral closures are important objects of study in commutative algebra and geometry [19].

A subset $C \subset X$ is a minimal vertex cover of the clutter $\mathcal{C}$ if: (i) every edge of $\mathcal{C}$ contains at least one vertex of $C$, and (ii) there is no proper subset of $C$ with the first property. If $C$ satisfies condition (i) only, then $C$ is called a vertex cover of $\mathcal{C}$. Let $C_{1}, \ldots, C_{s}$ be the minimal vertex covers of $\mathcal{C}$. The ideal of vertex covers of $\mathcal{C}$ is the square-free monomial ideal

$$
I_{c}(\mathcal{C})=\left(x^{u_{1}}, \ldots, x^{u_{s}}\right) \subset R,
$$


where $x^{u_{k}}=\prod_{x_{i} \in C_{k}} x_{i}$. The clutter associated to $I_{c}(\mathcal{C})$ is the blocker of $\mathcal{C}$, see [6]. Notice that the edges of the blocker are the minimal vertex covers of $\mathcal{C}$.

We now describe the content of the paper. A characterization of perfect graphs-in terms of Rees cones-is given (Proposition 2.2). We are able to prove that $R\left[I_{c}(G) t\right]$ is normal if $G$ is a perfect graph (Theorem 2.10) and that $R\left[I_{c}(G) t\right]$ is Gorenstein if $G$ is a perfect and unmixed graph (Corollary 2.12). To show the normality of $R\left[I_{c}(G) t\right]$, we study when the system $x \geq 0 ; x A \leq \mathbf{1}$ is TDI (Proposition 2.5), where TDI stands for Totally Dual Integral (see Section 2). If this system is TDI and the monomials in $F$ have the same degree, it is shown that $K[F t]$ is an Ehrhart ring (Proposition 2.7). This is one of the results that will be used in the proof of Theorem 2.10.

If $A$ is a balanced matrix, i.e., $A$ has no square submatrix of odd order with exactly two 1's in each row and column, and $J=I_{c}(\mathcal{C})$, then $R[I t]=R_{S}(I)$ and $R[J t]=$ $R_{S}(J)$, see [10]. We complement these results by showing that the Rees algebra of the dual $I^{*}$ of $I$ is normal if $A$ is balanced (Proposition 2.14).

By a result of Lyubeznik [16], $R_{S}(I(\mathcal{C}))$ is a $K$-algebra of finite type. Let $G$ be a graph. It is known that $R_{S}\left(I_{C}(G)\right)$ is generated as a $K$-algebra by monomials whose degree in $t$ is at most two [12, Theorem 5.1], and one may even give an explicit graph theoretical description of its minimal generators. Thus $R_{S}\left(I_{c}(G)\right)$ is well understood for graphs. In contrast, the minimal set of generators of $R_{S}(I(G))$ is very hard to describe in terms of $G$ (see [1]). If $G$ is a perfect graph we compute the integral Hilbert basis $\mathcal{H}$ of the Simis cone of $I(G)$ (see Definition 3.1 and Theorem 3.2). Then, using that $R_{S}(I(G))$ is the semigroup ring of $\mathbb{N} \mathcal{H}$ over $K$, we are able to prove that $R_{S}(I(G))$ is generated as a $K$-algebra by monomials associated to cliques of $G$ (Corollary 3.3).

Along the paper we introduce most of the notions that are relevant for our purposes. For unexplained terminology and notation we refer to [7, 14] and [3, 19]. See [6] for additional information about clutters and perfect graphs.

\section{Perfect graphs, cones, and Rees algebras}

We continue to use the notation and definitions used in the introduction. Let $\mathfrak{p}_{1}, \ldots, \mathfrak{p}_{s}$ be the minimal primes of $I(\mathcal{C})$ and let $C_{k}=\left\{x_{i} \mid x_{i} \in \mathfrak{p}_{k}\right\}$ be the minimal vertex cover of $\mathcal{C}$ that corresponds to $\mathfrak{p}_{k}$, see [20, Proposition 6.1.16]. There is a unique irreducible representation

$$
\mathbb{R}_{+}(I)=H_{e_{1}}^{+} \cap H_{e_{2}}^{+} \cap \cdots \cap H_{e_{n+1}}^{+} \cap H_{\ell_{1}}^{+} \cap H_{\ell_{2}}^{+} \cap \cdots \cap H_{\ell_{r}}^{+}
$$

such that each $\ell_{k}$ is in $\mathbb{Z}^{n+1}$, the non-zero entries of each $\ell_{k}$ are relatively prime, and none of the closed halfspaces $H_{e_{1}}^{+}, \ldots, H_{e_{n+1}}^{+}, H_{\ell_{1}}^{+}, \ldots, H_{\ell_{r}}^{+}$can be omitted from the intersection. Here $H_{a}^{+}$denotes the closed halfspace $H_{a}^{+}=\{x \mid\langle x, a\rangle \geq 0\}$ and $H_{a}$ stands for the hyperplane through the origin with normal vector $a$, where $\langle$, denotes the standard inner product. According to [9, Lemma 3.1] we may always assume that $\ell_{k}=-e_{n+1}+\sum_{x_{i} \in C_{k}} e_{i}$ for $1 \leq k \leq s$. We shall be interested in the irreducible representation of the Rees cone of the ideal of vertex covers of a perfect graph $G$ (see for instance Proposition 2.2). 
Let $G$ be a simple graph with vertex set $X=\left\{x_{1}, \ldots, x_{n}\right\}$. In what follows we shall always assume that $G$ has no isolated vertices. A colouring of the vertices of $G$ is an assignment of colours to the vertices of $G$ in such a way that adjacent vertices have distinct colours. The chromatic number of $G$ is the minimal number of colours in a colouring of $G$. A graph is perfect if for every induced subgraph $H$, the chromatic number of $H$ equals the size of the largest complete subgraph of $H$. The complement of $G$ is denoted by $G^{\prime}$. Recall that two vertices are adjacent in the graph $G$ if and only if they are not adjacent in the graph $G^{\prime}$.

Let $S$ be a subset of the vertices of $G$. The set $S$ is called independent if no two vertices of $S$ are adjacent. Notice the following duality: $S$ is a maximal independent set of $G$ (with respect to inclusion) if and only if $X \backslash S$ is a minimal vertex cover of $G$. We denote a complete subgraph of $G$ with $r$ vertices by $\mathcal{K}_{r}$. The empty set is regarded as an independent set whose incidence vector is the zero vector.

Theorem 2.1 ([14, Theorem 16.14]) The following statements are equivalent:

(a) $G$ is a perfect graph.

(b) The complement of $G$ is perfect.

(c) The independence polytope of $G$, i.e., the convex hull of the incidence vectors of the independent sets of $G$, is given by:

$$
\left\{\left(a_{i}\right) \in \mathbb{R}_{+}^{n} \mid \sum_{x_{i} \in \mathcal{K}_{r}} a_{i} \leq 1 ; \forall \mathcal{K}_{r} \subset G\right\} .
$$

Below we express the perfection of $G$ in terms of a Rees cone. The next result is just a dual reinterpretation of part (c) above, which is adequate to examine the normality and Gorensteiness of Rees algebras. We regard $\mathcal{K}_{0}$ as the empty set with zero elements. A sum over an empty set is defined to be 0 .

Proposition 2.2 Let $J=I_{c}(G)$ be the ideal of vertex covers of $G$. Then $G$ is perfect if and only if the following equality holds

$$
\mathbb{R}_{+}(J)=\left\{\left(a_{i}\right) \in \mathbb{R}^{n+1} \mid \sum_{x_{i} \in \mathcal{K}_{r}} a_{i} \geq(r-1) a_{n+1} ; \forall \mathcal{K}_{r} \subset G\right\} .
$$

Moreover this is the irreducible representation of $\mathbb{R}_{+}(J)$ if $G$ is perfect.

Proof $\Rightarrow$ ) The left hand side is contained in the right hand side because any minimal vertex cover of $G$ contains at least $r-1$ vertices of any $\mathcal{K}_{r}$. For the reverse inclusion take a vector $a=\left(a_{i}\right)$ satisfying $b=a_{n+1} \neq 0$ and

$$
\sum_{x_{i} \in \mathcal{K}_{r}} a_{i} \geq(r-1) b ; \forall \mathcal{K}_{r} \subset G \Longrightarrow \sum_{x_{i} \in \mathcal{K}_{r}}\left(a_{i} / b\right) \geq r-1 ; \forall \mathcal{K}_{r} \subset G .
$$

This implication follows because by making $r=0$ we get $b>0$. We may assume that $a_{i} \leq b$ for all $i$. Indeed if $a_{i}>b$ for some $i$, say $i=1$, then we can write $a=$ $e_{1}+\left(a-e_{1}\right)$. From the inequality

$$
\sum_{\substack{x_{i} \in \mathcal{K}_{r} \\ x_{1} \in \mathcal{K}_{r}}} a_{i}=a_{1}+\sum_{x_{i} \in \mathcal{K}_{r-1}} a_{i} \geq a_{1}+(r-2) b \geq 1+(r-1) b
$$


it is seen that $a-e_{1}$ belongs to the right hand side of Eq. (3). Thus, if necessary, we may apply this observation again to $a-e_{1}$ and so on till we get that $a_{i} \leq b$ for all $i$. Hence, by Theorem 2.1(c), the vector $\gamma=\mathbf{1}-\left(a_{1} / b, \ldots, a_{n} / b\right)$ belongs to the independence polytope of $G$. Thus we can write

$$
\gamma=\lambda_{1} w_{1}+\cdots+\lambda_{s} w_{s} ; \quad\left(\lambda_{i} \geq 0 ; \sum_{i} \lambda_{i}=1\right),
$$

where $w_{1}, \ldots, w_{s}$ are incidence vectors of independent sets of $G$. Hence

$$
\gamma=\lambda_{1}\left(\mathbf{1}-u_{1}^{\prime}\right)+\cdots+\lambda_{s}\left(\mathbf{1}-u_{s}^{\prime}\right)
$$

where $u_{1}^{\prime}, \ldots, u_{s}^{\prime}$ are incidence vectors of vertex covers of $G$. Since any vertex cover contains a minimal one, for each $i$ we can write $u_{i}^{\prime}=u_{i}+\epsilon_{i}$, where $u_{i}$ is the incidence vector of a minimal vertex cover of $G$ and $\epsilon_{i} \in\{0,1\}^{n}$. Therefore

$$
\begin{aligned}
& \mathbf{1}-\gamma=\lambda_{1} u_{1}^{\prime}+\cdots+\lambda_{s} u_{s}^{\prime} \Longrightarrow \\
& \quad a=b \lambda_{1}\left(u_{1}, 1\right)+\cdots+b \lambda_{s}\left(u_{s}, 1\right)+b \lambda_{1} \epsilon_{1}+\cdots+b \lambda_{s} \epsilon_{s} .
\end{aligned}
$$

Thus $a \in \mathbb{R}_{+}(J)$. If $b=0$, clearly $a \in \mathbb{R}_{+}(J)$. Hence we get equality in Eq. (3), as required. The converse follows using similar arguments.

To finish the proof it suffices to show that the set

$$
F=\left\{\left(a_{i}\right) \in \mathbb{R}^{n+1} \mid \sum_{x_{i} \in \mathcal{K}_{r}} a_{i}=(r-1) a_{n+1}\right\} \cap \mathbb{R}_{+}(J)
$$

is a facet of $\mathbb{R}_{+}(J)$. If $\mathcal{K}_{r}=\emptyset$, then $r=0$ and $F=H_{e_{n+1}} \cap \mathbb{R}_{+}(J)$, which is clearly a facet because $e_{1}, \ldots, e_{n} \in F$. If $r=1$, then $F=H_{e_{i}} \cap \mathbb{R}_{+}(J)$ for some $1 \leq i \leq n$, which is a facet because $e_{j} \in F$ for $j \notin\{i, n+1\}$ and there is at least one minimal vertex cover of $G$ not containing $x_{i}$. We may assume that $X^{\prime}=\left\{x_{1}, \ldots, x_{r}\right\}$ is the vertex set of $\mathcal{K}_{r}$ and $r \geq 2$. For each $1 \leq i \leq r$ there is a minimal vertex cover $C_{i}$ of $G$ not containing $x_{i}$. Notice that $C_{i}$ contains $X^{\prime} \backslash\left\{x_{i}\right\}$. Let $u_{i}$ be the incidence vector of $C_{i}$. Since the rank of $u_{1}, \ldots, u_{r}$ is $r$, it follows that the set

$$
\left\{\left(u_{1}, 1\right), \ldots,\left(u_{r}, 1\right), e_{r+1}, \ldots, e_{n}\right\}
$$

is a linearly independent set contained in $F$, i.e., $\operatorname{dim}(F)=n$. Hence $F$ is a facet of $\mathbb{R}_{+}(J)$ because the hyperplane that defines $F$ is a supporting hyperplane.

There are computer programs that determine the irreducible representation of a Rees cone [4]. Thus we may use Proposition 2.2 to determine whether a given graph is perfect, and in the process we may also determine its complete subgraphs. However this proposition is useful mainly for theoretical reasons. A direct consequence of this result (Lemma 2.3(b) below) will be used to prove one of our main results (Theorem 2.10).

Let $S$ be a set of vertices of a graph $G$. The induced subgraph $\langle S\rangle$ is the maximal subgraph of $G$ with vertex set $S$. A clique of a graph $G$ is a subset of the set of vertices that induces a complete subgraph. We will also call a complete subgraph of $G$ a clique. The support of $x^{a}=x_{1}^{a_{1}} \cdots x_{n}^{a_{n}}$ is $\operatorname{supp}\left(x^{a}\right)=\left\{x_{i} \mid a_{i}>0\right\}$. If $a_{i} \in\{0,1\}$ for all $i, x^{a}$ is called a square-free monomial. We regard the empty set as an independent set with zero elements. 
Lemma 2.3 (a) $I_{c}\left(G^{\prime}\right)=\left(\left\{x^{a} \mid X \backslash \operatorname{supp}\left(x^{a}\right)\right.\right.$ is a maximal clique of $\left.\left.G\right\}\right)$.

(b) If $G$ is perfect and $J^{\prime}=I_{c}\left(G^{\prime}\right)$, then $\mathbb{R}_{+}\left(J^{\prime}\right)$ is equal to

$$
\left\{\left(a_{i}\right) \in \mathbb{R}^{n+1} \mid \sum_{x_{i} \in S} a_{i} \geq(|S|-1) a_{n+1} ; \forall S \text { independent set of } G\right\} .
$$

Proof (a) Let $x^{a} \in R$ and let $S=\operatorname{supp}\left(x^{a}\right)$. Then $x^{a}$ is a minimal generator of $I_{c}\left(G^{\prime}\right)$ if and only if $S$ is a minimal vertex cover of $G^{\prime}$, which happens if and only if $X \backslash S$ is a maximal independent set of $G^{\prime}$, which in turn happens if and only if $\langle X \backslash S\rangle$ is a maximal complete subgraph of $G$. Thus the equality holds. (b) By Theorem 2.1 the graph $G^{\prime}$ is perfect. Hence the equality follows from Proposition 2.2.

Let $A$ be an integral matrix. The system $x \geq 0 ; x A \leq \mathbf{1}$ is called totally dual integral (TDI) if the minimum in the LP-duality equation

$$
\max \{\langle\alpha, x\rangle \mid x \geq 0 ; x A \leq \mathbf{1}\}=\min \{\langle y, \mathbf{1}\rangle \mid y \geq 0 ; A y \geq \alpha\}
$$

has an integral optimum solution $y$ for each integral vector $\alpha$ with finite minimum.

An incidence matrix $A$ of a clutter is called perfect if the polytope defined by the system $x \geq 0 ; x A \leq \mathbf{1}$ is integral, i.e., it has only integral vertices. The vertex-clique matrix of a graph $G$ is the $\{0,1\}$-matrix whose rows are indexed by the vertices of $G$ and whose columns are the incidence vectors of the maximal cliques of $G$.

Theorem 2.4 ([5, 15]) Let $A$ be the incidence matrix of a clutter. Then the following are equivalent:

(a) The system $x \geq 0 ; x A \leq \mathbf{1}$ is TDI.

(b) A is perfect.

(c) A is the vertex-clique matrix of a perfect graph.

Proposition 2.5 Let $A$ be an $n \times q$ matrix with entries in $\mathbb{N}$ and let $v_{1}, \ldots, v_{q}$ be its column vectors. Then the system $x \geq 0 ; x A \leq \mathbf{1}$ is TDI if and only if

(i) the polyhedron $\{x \mid x \geq 0 ; x A \leq \mathbf{1}\}$ is integral, and

(ii) $\mathbb{R}_{+} \mathcal{B} \cap \mathbb{Z}^{n+1}=\mathbb{N} \mathcal{B}$, where $\mathcal{B}=\left\{\left(v_{1}, 1\right), \ldots,\left(v_{q}, 1\right),-e_{1}, \ldots,-e_{n}\right\}$.

Proof $\Rightarrow$ ) By [17, Corollary 22.1c] we get that (i) holds. To prove (ii) take $(\alpha, b) \in$ $\mathbb{R}_{+} \mathcal{B} \cap \mathbb{Z}^{n+1}$, where $\alpha \in \mathbb{Z}^{n}$ and $b \in \mathbb{Z}$. By hypothesis the minimum in Eq. (4) has an integral optimum solution $y=\left(y_{i}\right)$ such that $|y|=\langle y, \mathbf{1}\rangle \leq b$. Since $y \geq 0$ and $\alpha \leq A y$ we can write

$$
\begin{gathered}
\alpha=y_{1} v_{1}+\cdots+y_{q} v_{q}-\delta_{1} e_{1}-\cdots-\delta_{n} e_{n} \quad\left(\delta_{i} \in \mathbb{N}\right) \Longrightarrow \\
(\alpha, b)=y_{1}\left(v_{1}, 1\right)+\cdots+y_{q-1}\left(v_{q-1}, 1\right)+\left(y_{q}+b-|y|\right)\left(v_{q}, 1\right)-(b-|y|) v_{q}-\delta,
\end{gathered}
$$

where $\delta=\left(\delta_{i}\right)$. As the entries of $A$ are in $\mathbb{N}$, the vector $-v_{q}$ can be written as a nonnegative integer combination of $-e_{1}, \ldots,-e_{n}$. Thus $(\alpha, b) \in \mathbb{N} \mathcal{B}$. This proves (ii).

$\Leftarrow$ ) Assume that the system $x \geq 0 ; x A \leq \mathbf{1}$ is not TDI. Then there exists an $\alpha_{0} \in \mathbb{Z}^{n}$ such that if $y_{0}$ is an optimal solution of the linear program:

$$
\min \left\{\langle y, \mathbf{1}\rangle \mid y \geq 0 ; A y \geq \alpha_{0}\right\},
$$


then $y_{0}$ is not integral. We claim that also the optimal value $\left|y_{0}\right|=\left\langle y_{0}, \mathbf{1}\right\rangle$ of this linear program is not integral. If $\left|y_{0}\right|$ is integral, then $\left(\alpha_{0},\left|y_{0}\right|\right)$ is in $\mathbb{Z}^{n+1} \cap \mathbb{R}_{+} \mathcal{B}$. Hence by (ii), we get that $\left(\alpha_{0},\left|y_{0}\right|\right)$ is in $\mathbb{N} \mathcal{B}$, but this readily yields that the linear program of Eq. (5) has an integral optimal solution, a contradiction. This completes the proof of the claim. Consider the dual linear program:

$$
\max \left\{\left\langle x, \alpha_{0}\right\rangle \mid x \geq 0, x A \leq \mathbf{1}\right\}
$$

Its optimal value is attained at a vertex $x_{0}$ of $\{x \mid x \geq 0 ; x A \leq \mathbf{1}\}$. Then by LP duality we get $\left\langle x_{0}, \alpha_{0}\right\rangle=\left|y_{0}\right| \notin \mathbb{Z}$. Hence $x_{0}$ is not integral, a contradiction to the integrality of $\{x \mid x \geq 0 ; x A \leq \mathbf{1}\}$.

Remark 2.6 If $A$ is a matrix with entries in $\mathbb{Z}$ satisfying (i) and (ii), then the system $x \geq 0 ; x A \leq \mathbf{1}$ is TDI.

Let $v_{1}, \ldots, v_{q}$ be a set of points in $\mathbb{N}^{n}$ and let $P=\operatorname{conv}\left(v_{1}, \ldots, v_{q}\right)$. The Ehrhart ring of the lattice polytope $P$ is the $K$-subring of $R[t]$ given by

$$
A(P)=K\left[\left\{x^{a} t^{b} \mid a \in b P \cap \mathbb{Z}^{n}\right\}\right] .
$$

Proposition 2.7 Let $A$ be a perfect matrix with column vectors $v_{1}, \ldots, v_{q}$. If there is $x_{0} \in \mathbb{R}^{n}$ such that all the entries of $x_{0}$ are positive and $\left\langle v_{i}, x_{0}\right\rangle=1$ for all $i$, then $A(P)=K\left[x^{v_{1}} t, \ldots, x^{v_{q}} t\right]$.

Proof Let $x^{a} t^{b} \in A(P)$. Then we can write $(a, b)=\sum_{i=1}^{q} \lambda_{i}\left(v_{i}, 1\right)$, where $\lambda_{i} \geq 0$ for all $i$. Hence $\left\langle a, x_{0}\right\rangle=b$. By Theorem 2.4 the system $x \geq 0 ; x A \leq \mathbf{1}$ is TDI. Hence applying Proposition 2.5(ii) we have:

$$
(a, b)=\eta_{1}\left(v_{1}, 1\right)+\cdots+\eta_{q}\left(v_{q}, 1\right)-\delta_{1} e_{1}-\cdots-\delta_{n} e_{n} \quad\left(\eta_{i} \in \mathbb{N} ; \delta_{i} \in \mathbb{N}\right) .
$$

Consequently $b=\left\langle a, x_{0}\right\rangle=b-\delta_{1}\left\langle x_{0}, e_{1}\right\rangle-\cdots-\delta_{n}\left\langle x_{0}, e_{n}\right\rangle$. Using that $\left\langle x_{0}, e_{i}\right\rangle>0$ for all $i$, we conclude that $\delta_{i}=0$ for all $i$, i.e., $x^{a} t^{b} \in K\left[x^{v_{1}} t, \ldots, x^{v_{q}} t\right]$.

Recall that the clutter $\mathcal{C}$ (or the edge ideal $I(\mathcal{C})$ ) is called unmixed if all the minimal vertex covers of $\mathcal{C}$ have the same cardinality.

Corollary 2.8 If $G$ is a perfect unmixed graph and $v_{1}, \ldots, v_{q}$ are the incidence vectors of the maximal independent sets of $G$, then $K\left[x^{v_{1}} t, \ldots, x^{v_{q}} t\right]$ is normal.

Proof The minimal vertex covers of $G$ are exactly the complements of the maximal independent sets of $G$. Thus $\left|v_{i}\right|=d$ for all $i$, where $d=\operatorname{dim}(R / I(G))$. On the other hand the maximal independent sets of $G$ are exactly the maximal cliques of $G^{\prime}$. Thus, by Theorem 2.4 and Proposition 2.7, the subring $K\left[x^{v_{1}} t, \ldots, x^{v_{q}} t\right]$ is an Ehrhart ring, and consequently it is normal.

Let $\mathcal{C}$ be a clutter and let $A$ be its incidence matrix. The clutter $\mathcal{C}$ satisfies the max-flow min-cut (MFMC) property if both sides of the LP-duality equation

$$
\min \{\langle\alpha, x\rangle \mid x \geq 0 ; x A \geq \mathbf{1}\}=\max \{\langle y, \mathbf{1}\rangle \mid y \geq 0 ; A y \leq \alpha\}
$$


have integral optimum solutions $x$ and $y$ for each non-negative integral vector $\alpha$, see [6]. Let $I$ be the edge ideal of $\mathcal{C}$. Closely related to $\mathbb{R}_{+}(I)$ is the set covering polyhedron:

$$
Q(A)=\left\{x \in \mathbb{R}^{n} \mid x \geq 0, x A \geq \mathbf{1}\right\},
$$

see [10, Theorem 3.1]. Its integral vertices are precisely the incidence vectors of the minimal vertex covers of $\mathcal{C}[10$, Proposition 2.2].

Corollary 2.9 Let $\mathcal{C}$ be a clutter and let $A$ be its incidence matrix. If all the edges of $\mathcal{C}$ have the same cardinality and the polyhedra

$$
\{x \mid x \geq 0 ; x A \leq \mathbf{1}\} \text { and }\{x \mid x \geq 0 ; x A \geq \mathbf{1}\}
$$

are integral, then $\mathcal{C}$ has the max-flow min-cut property.

Proof By [10, Proposition 4.4 and Theorem 4.6] we have that $\mathcal{C}$ has the max-flow min-cut property if and only if $Q(A)$ is integral and $K\left[x^{v_{1}} t, \ldots, x^{v_{q}} t\right]=A(P)$, where $v_{1}, \ldots, v_{q}$ are the column vectors of $A$ and $P=\operatorname{conv}\left(v_{1}, \ldots, v_{q}\right)$. Thus the result follows from Proposition 2.7.

The clique clutter of a graph $G$, denoted by $\operatorname{cl}(G)$, is the clutter on $V(G)$ whose edges are the maximal cliques of $G$.

Theorem 2.10 If $G$ is a perfect graph, then $R\left[I_{c}(G) t\right]$ is normal.

Proof Let $G^{\prime}$ be the complement of $G$ and let $J^{\prime}=I_{c}\left(G^{\prime}\right)$. Since $G^{\prime}$ is perfect it suffices to prove that $R\left[J^{\prime} t\right]$ is normal.

Case (A): Assume that all the maximal cliques of $G$ have the same number of elements. Let $F=\left\{x^{v_{1}}, \ldots, x^{v_{q}}\right\}$ be the set of monomials of $R$ whose support is a maximal clique of $G$. We set $F^{\prime}=\left\{x^{w_{1}}, \ldots, x^{w_{q}}\right\}$, where $x^{w_{i}}=x_{1} \cdots x_{n} / x^{v_{i}}$. By Lemma 2.3(a) we have $J^{\prime}=\left(F^{\prime}\right)$. Consider the matrices

$$
B=\left(\begin{array}{ccc}
v_{1} & \cdots & v_{q} \\
1 & \cdots & 1
\end{array}\right) \quad \text { and } \quad B^{\prime}=\left(\begin{array}{ccc}
w_{1} & \cdots & w_{q} \\
1 & \cdots & 1
\end{array}\right),
$$

where the $v_{i}$ 's and $w_{j}$ 's are regarded as column vectors. Using the last row of $B$ as a pivot it is seen that $B$ is equivalent over $\mathbb{Z}$ to $B^{\prime}$. Let $A$ be the incidence matrix of $\operatorname{cl}(G)$, the clique clutter of $G$, whose columns are $v_{1}, \ldots, v_{q}$. As the matrix $A$ is perfect, by Proposition 2.7, we obtain that $K[F t]=A(P)$, where $A(P)$ is the Ehrhart ring of $P=\operatorname{conv}\left(v_{1}, \ldots, v_{q}\right)$. In particular $K[F t]$ is normal because Ehrhart rings are normal. According to [8, Theorem 3.9] we have that $K[F t]=A(P)$ if and only if $K[F t]$ is normal and $B$ diagonalizes over $\mathbb{Z}$ to an "identity" matrix. Consequently the matrix $B^{\prime}$ diagonalizes to an identity matrix along with $B$. Since the rings $K\left[F^{\prime} t\right]$ and $K[F t]$ are isomorphic, we get that $K\left[F^{\prime} t\right]$ is normal. Thus, again by [8, Theorem 3.9], we obtain the equality $K\left[F^{\prime} t\right]=A\left(P^{\prime}\right)$, where $A\left(P^{\prime}\right)$ is the Ehrhart ring of $P^{\prime}=\operatorname{conv}\left(w_{1}, \ldots, w_{q}\right)$. Let $H_{a}^{+}$be any of the halfspaces that occur in the irreducible representation of the Rees cone $\mathbb{R}_{+}\left(J^{\prime}\right)$. By Lemma 2.3(b) 
the first $n$ entries of $a$ are either 0 or 1. Hence by [10, Proposition 4.2] we get the equality

$$
A\left(P^{\prime}\right)\left[x_{1}, \ldots, x_{n}\right]=\overline{R\left[J^{\prime} t\right]} .
$$

Therefore $R\left[J^{\prime} t\right]=K\left[F^{\prime} t\right]\left[x_{1}, \ldots, x_{n}\right]=A\left(P^{\prime}\right)\left[x_{1}, \ldots, x_{n}\right]=\overline{R\left[J^{\prime} t\right]}$, that is, $R\left[J^{\prime} t\right]$ is normal.

Case (B): Assume that not all the maximal cliques of $G$ have the same number of elements. Let $C$ be a maximal clique of $G$ of lowest size and let $w$ be its incidence vector. For simplicity of notation assume that $C=\left\{x_{1}, \ldots, x_{r}\right\}$. Let $z=x_{n+1} \notin V(G)$ be a new vertex. We construct a new graph $H$ as follows. Its vertex set is $V(H)=$ $V(G) \cup\{z\}$ and its edge set is

$$
E(H)=E(G) \cup\left\{\left\{z, x_{1}\right\}, \ldots,\left\{z, x_{r}\right\}\right\} .
$$

Notice that $C \cup\{z\}$ is the only maximal clique of $H$ containing $z$. Thus it is seen that the edges of the clique clutter of $H$ are related to those of the clique clutter of $G$ as follows:

$$
E(\operatorname{cl}(H))=(E(\operatorname{cl}(G)) \backslash\{C\}) \cup\{C \cup\{z\}\} .
$$

From the proof of [7, Proposition 5.5.2] it follows that if we paste together $G$ and the complete subgraph induced by $C \cup\{z\}$ along the complete subgraph induced by $C$ we obtain a perfect graph, i.e., $H$ is perfect. This construction is different from the famous Lovász replication of a vertex, as explained in [6, Lemma 3.3]. The contraction of $\operatorname{cl}(H)$ at $z$, denoted by $\operatorname{cl}(H) / z$, is the clutter of minimal elements of $\{S \backslash\{z\} \mid S \in \operatorname{cl}(H)\}$. In our case we have $\operatorname{cl}(H) / z=\operatorname{cl}(G)$, i.e., $\operatorname{cl}(G)$ is a minor of $\operatorname{cl}(H)$ obtained by contraction. By successively adding new vertices $z_{1}=z, z_{2}, \ldots, z_{r}$, following the construction above, we obtain a perfect graph $H$ whose maximal cliques have the same size and such that $\operatorname{cl}(G)$ is a minor of $\operatorname{cl}(H)$ obtained by contraction of the vertices $z_{1}, \ldots, z_{s}$. By case (A) we obtain that the ideal $L=I_{c}\left(H^{\prime}\right)$ of minimal vertex covers of $H^{\prime}$ is normal. Since $L$ is generated by all the square-free monomials $m$ of $R\left[z_{1}, \ldots, z_{s}\right]$ such that $V(H) \backslash \operatorname{supp}(m)$ is a maximal clique of $H$, it follows that $J^{\prime}$ is obtained from $L$ by making $z_{i}=1$ for all $i$. Hence $R\left[J^{\prime} t\right]$ is normal because the normality property of Rees algebras of edge ideals is closed under taking minors [9, Proposition 4.3].

Example 2.11 If $G$ is a pentagon, then the Rees algebra of $I_{c}(G)$ is normal and $G$ is not perfect.

Corollary 2.12 If $G$ is perfect and unmixed, then $R\left[I_{c}(G) t\right]$ is a Gorenstein standard graded $K$-algebra.

Proof Let $g$ be the height of the edge ideal $I(G)$ and let $J=I_{c}(G)$. By assigning $\operatorname{deg}\left(x_{i}\right)=1$ and $\operatorname{deg}(t)=-(g-1)$, the Rees algebra $R[J t]$ becomes a graded $K$ algebra generated by monomials of degree 1 . The Rees ring $R[\mathrm{Jt}]$ is a normal domain by Theorem 2.10. Then according to a formula of Danilov-Stanley [3, Theorem 6.3.5] its canonical module is the ideal of $R[\mathrm{~J} t]$ given by

$$
\omega_{R[J t]}=\left(\left\{x_{1}^{a_{1}} \cdots x_{n}^{a_{n}} t^{a_{n+1}} \mid a=\left(a_{i}\right) \in \mathbb{R}_{+}(J)^{\mathrm{o}} \cap \mathbb{Z}^{n+1}\right\}\right),
$$


where $\mathbb{R}_{+}(J)^{\mathrm{o}}$ denotes the topological interior of the Rees cone of $J$. By a result of Hochster [13] the ring $R[J t]$ is Cohen-Macaulay. Using Eq. (3) it is seen that the vector $(1, \ldots, 1)$ is in the interior of the Rees cone, i.e., $x_{1} \cdots x_{n} t$ belongs to $\omega_{R[J t]}$. Take an arbitrary monomial $x^{a} t^{b}=x_{1}^{a_{1}} \cdots x_{n}^{a_{n}} t^{b}$ in the ideal $\omega_{R[J t]}$, that is $(a, b) \in \mathbb{R}_{+}(J)^{\mathrm{o}}$. Hence the vector $(a, b)$ has positive integer entries and satisfies

$$
\sum_{x_{i} \in \mathcal{K}_{r}} a_{i} \geq(r-1) b+1
$$

for every complete subgraph $\mathcal{K}_{r}$ of $G$. If $b=1$, clearly $x^{a} t^{b}$ is a multiple of $x_{1} \cdots x_{n} t$. Now assume $b \geq 2$. Using the normality of $R[J t]$ and Eqs. (3) and (6) it follows that the monomial $m=x_{1}^{a_{1}-1} \cdots x_{n}^{a_{n}-1} t^{b-1}$ belongs to $R[J t]$. Since $x^{a} t^{b}=m x_{1} \cdots x_{n} t$, we obtain that $\omega_{R[J t]}$ is generated by $x_{1} \cdots x_{n} t$ and thus $R[\mathrm{Jt}]$ is a Gorenstein ring.

A graph $G$ is chordal if every cycle of $G$ of length $n \geq 4$ has a chord. A chord of a cycle is an edge joining two non adjacent vertices of the cycle.

Corollary 2.13 If $J$ is a Cohen-Macaulay square-free monomial ideal of height two, then $R[J t]$ is normal.

Proof Consider the graph $G$ whose edges are the pairs $\left\{x_{i}, x_{j}\right\}$ such that $\left(x_{i}, x_{j}\right)$ is a minimal prime of $J$. Notice that $J=I_{c}(G)$. By [20, Theorem 6.7.13], the ideal $I_{C}(G)$ is Cohen-Macaulay if and only if $G^{\prime}$ is a chordal graph. Since chordal graphs are perfect [7, Proposition 5.5.2], we obtain that $G^{\prime}$ is perfect. Thus $G$ is a perfect graph by Theorem 2.1. Applying Theorem 2.10 we conclude that $R[J t]$ is normal.

Recall that a matrix with $\{0,1\}$-entries is called balanced if $A$ has no square submatrix of odd order with exactly two 1's in each row and column,

Proposition 2.14 Let $A$ be a $\{0,1\}$-matrix with column vectors $v_{1}, \ldots, v_{q}$ and let $w_{i}=\mathbf{1}-v_{i}$. If $A$ is balanced, then the Rees algebra of $I^{*}=\left(x^{w_{1}}, \ldots, x^{w_{q}}\right)$ is a normal domain.

Proof According to [2], [18, Corollary 83.1a(vii), p. 1441] $A$ is balanced if and only if every submatrix of $A$ is perfect. By adjoining rows of unit vectors to $A$ and since the normality property of edge ideals is closed under taking minors [9, Proposition 4.3] we may assume that $\left|v_{i}\right|=d$ for all $i$. By Theorem 2.4 there is a perfect graph $G$ such that $A$ is the vertex-clique matrix of $G$. Thus following the first part of the proof of Theorem 2.10 , we obtain that $R\left[I^{*} t\right]$ is normal.

Consider the ideals $I=\left(x^{v_{1}}, \ldots, x^{v_{q}}\right)$ and $I^{*}=\left(x^{w_{1}}, \ldots, x^{w_{q}}\right)$. Following the terminology of matroid theory we call $I^{*}$ the dual of $I$. Notice the following duality. If $A$ is the vertex-clique matrix of a graph $G$, then $I^{*}$ is precisely the ideal of vertex covers of $G^{\prime}$. 


\section{Symbolic Rees algebras of edge ideals}

Let $G$ be a graph with vertex set $X=\left\{x_{1}, \ldots, x_{n}\right\}$ and let $I=I(G)$ be its edge ideal [20, Chapter 6]. The main purpose of this section is to study the symbolic Rees algebra of $I$ and the Simis cone of $I$ when $G$ is a perfect graph. We show that the cliques of a perfect graph $G$ completely determine both the Hilbert basis of the Simis cone and the symbolic Rees algebra of $I(G)$.

Definition 3.1 Let $C_{1}, \ldots, C_{s}$ be the minimal vertex covers of $G$. The symbolic Rees cone or Simis cone of $I$ is the rational polyhedral cone:

$$
\operatorname{Cn}(I)=H_{e_{1}}^{+} \cap \cdots \cap H_{e_{n+1}}^{+} \cap H_{\left(u_{1},-1\right)}^{+} \cap \cdots \cap H_{\left(u_{s},-1\right)}^{+},
$$

where $u_{k}=\sum_{x_{i} \in C_{k}} e_{i}$ for $1 \leq k \leq s$.

Simis cones were introduced in [9] to study symbolic Rees algebras of square-free monomial ideals. If $\mathcal{H}$ is an integral Hilbert basis of $\mathrm{Cn}(I)$, then $R_{S}(I(G))$ equals $K[\mathbb{N} \mathcal{H}]$, the semigroup ring of $\mathbb{N} \mathcal{H}$ (see [9]). This result is interesting because it allows us to compute the minimal generators of $R_{S}(I(G))$ using Hilbert basis. Next we describe $\mathcal{H}$ when $G$ is perfect.

Theorem 3.2 Let $\omega_{1}, \ldots, \omega_{p}$ be the incidence vectors of the non-empty cliques of a perfect graph $G$ and let

$$
\mathcal{H}=\left\{\left(\omega_{1},\left|\omega_{1}\right|-1\right), \ldots,\left(\omega_{p},\left|\omega_{p}\right|-1\right)\right\} .
$$

Then $\mathbb{N} \mathcal{H}=\operatorname{Cn}(I) \cap \mathbb{Z}^{n+1}$, where $\mathbb{N} \mathcal{H}$ is the subsemigroup of $\mathbb{N}^{n+1}$ generated by $\mathcal{H}$, that is, $\mathcal{H}$ is the integral Hilbert basis of $\mathrm{Cn}(I)$.

Proof The inclusion $\mathbb{N H} \subset \operatorname{Cn}(I) \cap \mathbb{Z}^{n+1}$ is clear because each clique of size $r$ intersects any minimal vertex cover in at least $r-1$ vertices. Let us show the reverse inclusion. Let $(a, b)$ be a minimal generator of $\operatorname{Cn}(I) \cap \mathbb{Z}^{n+1}$, where $0 \neq a=\left(a_{i}\right) \in \mathbb{N}^{n}$ and $b \in \mathbb{N}$. Then

$$
\sum_{x_{i} \in C_{k}} a_{i}=\left\langle a, u_{k}\right\rangle \geq b,
$$

for all $k$. If $b=0$ or $b=1$, then $(a, b)=e_{i}$ for some $i \leq n$ or $(a, b)=\left(e_{i}+e_{j}, 1\right)$ for some edge $\left\{x_{i}, x_{j}\right\}$ respectively. In both cases $(a, b) \in \mathcal{H}$. Thus we may assume that $b \geq 2$ and $a_{j} \geq 1$ for some $j$. Using Eq. (7) we obtain

$$
\sum_{x_{i} \in C_{k}} a_{i}+\sum_{x_{i} \in X \backslash C_{k}} a_{i}=|a| \geq b+\sum_{x_{i} \in X \backslash C_{k}} a_{i}=b+\left\langle\mathbf{1}-u_{k}, a\right\rangle,
$$

for all $k$, where $X=\left\{x_{1}, \ldots, x_{n}\right\}$ is the vertex set of $G$. Set $c=|a|-b$. Notice that $c \geq 1$ because $a \neq 0$. Indeed if $c=0$, from Eq. (8) we get $\sum_{x_{i} \in X \backslash C_{k}} a_{i}=0$ for all $k$, i.e., $a=0$, a contradiction. Consider the vertex-clique matrix of $G^{\prime}$ :

$$
A^{\prime}=\left(\mathbf{1}-u_{1} \cdots \mathbf{1}-u_{s}\right)
$$


where $\mathbf{1}-u_{1}, \ldots, \mathbf{1}-u_{s}$ are regarded as column vectors. From Eq. (8) we get $(a / c) A^{\prime} \leq 1$. Hence by Theorem 2.1(c) we obtain that $a / c$ belongs to $\operatorname{conv}\left(\omega_{0}, \omega_{1}, \ldots, \omega_{p}\right)$, where $\omega_{0}=0$, i.e., we can write $a / c=\lambda_{0} \omega_{0}+\cdots+\lambda_{p} \omega_{p}$, where $\lambda_{i} \geq 0$ for all $i$ and $\sum_{i} \lambda_{i}=1$. Thus we can write

$$
(a, c)=c \lambda_{0}\left(\omega_{0}, 1\right)+\cdots+c \lambda_{p}\left(\omega_{p}, 1\right) .
$$

Using Theorem 2.4(a) it is not hard to see that the subring $K\left[\left\{x^{\omega_{i}} t \mid 0 \leq i \leq p\right\}\right]$ is normal. Hence there are $\eta_{0}, \ldots, \eta_{p}$ in $\mathbb{N}$ such that

$$
(a, c)=\eta_{0}\left(\omega_{0}, 1\right)+\cdots+\eta_{p}\left(\omega_{p}, 1\right) .
$$

Thus $|a|=\eta_{0}\left|\omega_{0}\right|+\cdots+\eta_{p}\left|\omega_{p}\right|$ and $c=\eta_{0}+\cdots+\eta_{p}=|a|-b$, consequently:

$$
(a, b)=\eta_{0}\left(\omega_{0},\left|\omega_{0}\right|-1\right)+\eta_{1}\left(\omega_{1},\left|\omega_{1}\right|-1\right)+\cdots+\eta_{p}\left(\omega_{p},\left|\omega_{p}\right|-1\right) .
$$

Notice that there is $u_{\ell}$ such that $\left\langle a, u_{\ell}\right\rangle=b$; otherwise since $a_{j} \geq 1$, by Eq. (7) the vector $(a, b)-e_{j}$ would be in $\operatorname{Cn}(I) \cap \mathbb{Z}^{n+1}$, contradicting the minimality of $(a, b)$. Therefore from the equality

$$
0=\left\langle(a, b),\left(u_{\ell},-1\right)\right\rangle=\eta_{0}+\sum_{i=1}^{p} \eta_{i}\left\langle\left(\omega_{i},\left|\omega_{i}\right|-1\right),\left(u_{\ell},-1\right)\right\rangle
$$

we conclude that $\eta_{0}=0$, i.e., $(a, b) \in \mathbb{N} \mathcal{H}$, as required.

Corollary 3.3 If $G$ is a perfect graph, then

$$
R_{S}(I(G))=K\left[x^{a} t^{r} \mid x^{a} \text { is square-free; }\left\langle\operatorname{supp}\left(x^{a}\right)\right\rangle=\mathcal{K}_{r+1} ; 0 \leq r<n\right] .
$$

Proof Let $K[\mathbb{N H}]$ be the semigroup ring with coefficients in $K$ of the semigroup $\mathbb{N} \mathcal{H}$. By [9, Theorem 3.5] we have the equality $R_{S}(I(G))=K[\mathbb{N} \mathcal{H}]$, thus the formula follows from Theorem 3.2.

Corollary 3.4 ([1]) If $G$ is a complete graph, then

$$
R_{S}(I(G))=K\left[x^{a} t^{r} \mid x^{a} \text { is square-free; } \operatorname{deg}\left(x^{a}\right)=r+1 ; r \geq 0\right] .
$$

\section{References}

1. Bahiano, C.: Symbolic powers of edge ideals. J. Algebra 273(2), 517-537 (2004)

2. Berge, C.: Balanced matrices. Math. Program. 2(1), 19-31 (1972)

3. Bruns, W., Herzog, J.: Cohen-Macaulay Rings. Cambridge University Press, Cambridge (1997), Revised edition

4. Bruns, W., Koch, R.: NormaLiZ, computing normalizations of affine semigroups (2003). Available from: ftp.mathematik.Uni-Osnabrueck.DE/pub/osm/kommalg/software

5. Chvátal, V.: On certain polytopes associated with graphs. J. Comb. Theory Ser. B 18, 138-154 (1975)

6. Cornuéjols, G.: Combinatorial Optimization: Packing and Covering. CBMS-NSF Regional Conference Series in Applied Mathematics, vol. 74, SIAM (2001)

7. Diestel, R.: Graph Theory, 2nd edn. Graduate Texts in Mathematics, vol. 173. Springer, New York (2000) 
8. Escobar, C., Martínez-Bernal, J., Villarreal, R.H.: Relative volumes and minors in monomial subrings. Linear Algebra Appl. 374, 275-290 (2003)

9. Escobar, C., Villarreal, R.H., Yoshino, Y.: Torsion freeness and normality of blowup rings of monomial ideals. In: Commutative Algebra. Lecture Notes Pure Appl. Math., vol. 244, pp. 69-84. Chapman \& Hall/CRC, Boca Raton (2006)

10. Gitler, I., Reyes, E., Villarreal, R.H.: Blowup algebras of square-free monomial ideals and some links to combinatorial optimization problems. Rocky Mt. J. Math. 37(4) (2007, to appear)

11. Gitler, I., Valencia, C., Villarreal, R.H.: A note on Rees algebras and the MFMC property. Beiträge Algebra Geom. 48(1), 141-150 (2007)

12. Herzog, J., Hibi, T., Trung, N.V.: Symbolic powers of monomial ideals and vertex cover algebras. Adv. Math. 210, 304-322 (2007)

13. Hochster, M.: Rings of invariants of tori, Cohen-Macaulay rings generated by monomials, and polytopes. Ann. Math. 96, 318-337 (1972)

14. Korte, B., Vygen, J.: Combinatorial Optimization Theory and Algorithms. Springer, New York (2000)

15. Lovász, L.: Normal hypergraphs and the perfect graph conjecture. Discret. Math. 2(3), 253-267 (1972)

16. Lyubeznik, G.: On the arithmetical rank of monomial ideals. J. Algebra 112, 86-89 (1988)

17. Schrijver, A.: Theory of Linear and Integer Programming. Wiley, New York (1986)

18. Schrijver, A.: Combinatorial Optimization. Algorithms and Combinatorics, vol. 24. Springer, Berlin (2003)

19. Vasconcelos, W.V.: Integral Closure. Springer Monographs in Mathematics. Springer, New York (2005)

20. Villarreal, R.H.: Monomial Algebras. Dekker, New York (2001) 